\title{
The Informational Basis of Social Judgments: Operations in Forming an Impression of Another Person
}

\author{
Eugene Burnstein and YaAcov Schul
}

The University of Michigan

Received October 7, 1980

\begin{abstract}
This paper describes four functionally distinct operations carried out in forming an impression of another person: Initial encoding, elaborative encoding, integration, and decision. These processes were identified empirically on the basis of their differential sensitivity to the amount and the consistency of information in a trait description. In Study 1 as the trait set increased in size, processing was hindered to the greatest extent during initial encoding, to a moderate extent during elaborative encoding, and least during the integration-decision interval. Varying consistency produced the opposite pattern of results, that is, an inconsistent trait description hindered processing the least during initial encoding, a moderate amount during elaborative encoding, and the most during the integration-decision interval. In Study 2 the quantity of information was manipulated via implicit knowledge, that is, the associative structure activated by the trait (rather than set size). Because implicit information is "preintegrated," implication-rich traits did not hinder initial or elaborative encoding more than implication-poor traits. The decision operation, however, was performed more rapidly with implication-rich traits than with implication-poor ones, which strongly suggests that once information is integrated, the "richer" the representation, the easier it is to arrive at a decision.
\end{abstract}

Traditionally, research on social judgments in general, and impression formation in particular, has been concerned with the functional relationship between properties of the judgment (e.g., the extremity of the impression) and properties of the stimulus information (e.g., the number of traits, their consistency). Until a few years ago there had been relatively little explication of the mental operations involved in forming such opinions. However, because of recent theoretical and methodological developments in cognitive psychology, considerable effort is now being made to analyze these operations (Carlston, 1980; Ebbesen, 1980;

The research reported here was supported by a grant from National Science Foundation (BNS 7915416). Address reprint requests to Eugene Burnstein, Research Center for Group Dynamics, Institute for Social Research, University of Michigan, Ann Arbor, MI 48106. 
Hastie \& Carlston, 1980; Srull \& Wyer, 1979; Wyer \& Carlston, 1979). The present research continues this line of analysis in attempting to isolate the processes underlying impression formation by chronometric methods.

We propose to divide the mental operations involved in impression formation into four classes: initial encoding, elaborative encoding, integration, and decision. We will focus on the latter two since they are unique to impression formation (see below). Initial and elaborative encoding primarily will serve as baseline operations for specifying those functions peculiar to integration and decision. For convenience of presentation each class of operations is referred to as a single process. Whether the above partition is in fact meaningful will be shown by the differential sensitivity of these processes to features of the stimulus information. More specifically, individuals will be presented with sets of traits that vary in size (i.e., number of traits) and affective consistency (i.e., all positive or negative, or mixed). The individuals will then perform an orienting task that involves one (or more) of the above operations (cf. Craik \& Tulving, 1975).

During initial encoding the external stimulus undergoes perceptual analysis and segmentation (Newtson, 1973; Ebbesen, 1980) whereby it is transformed into symbolic structures-the memory codes (e.g., Posner, 1978). Functionally, these operations associate a separate memory code with each piece of information in the external stimulus. In the present studies initial encoding will be induced by an orienting task that involves physical matching (Posner \& Keele, 1967). That is to say, a set of traits are shown on a video monitor and individuals indicate when they have finished reading them. Immediately following this, the traits are replaced by a probe (a single trait) and individuals indicate whether or not the probe appeared in the previous set. The time needed to read a set is taken as a measure of the processing required to prepare the traits for physical matching. The operations involved in this preparation are assumed to correspond to those of initial encoding, that is, each piece of information is encoded separately with minimal semantic analysis, perhaps none beyond what is performed automatically whenever such codes are activated by semantic material [the Stroop effect (Dyer, 1973) is a good example of such a phenomenon; also, cf., Posner, 1972 for a detailed discussion of automatic activation of one type of code by another]. This suggests that initial encoding ought to be most sensitive to features associated with the separate elements in the set (c.g., the number of traits), and least sensitive to relationships between these elements (e.g., their consistency).

Once information has made contact with memory, elaborative encoding may occur. This involves linking the pieces of information extracted from the external stimulus with prior knowledge. Prior knowledge is 
assumed to be represented in the form of schemata. Each schema is conceived as a set of propositions that describe either some prototypical series of events (i.e., a script, in Schank \& Abelson, 1977) or objects (i.e., a frame in Minsky, 1975; Friedman, 1979). The knowledge represented within a schema serves two purposes: First, it specifies which stimuli can be matched to or instantiate the schema. Second, when instantiation occurs, information in the stimulus is supplemented by knowledge contained in the schema. The latter is said to be knowledge introduced by default; that is, it specifies those features that typically occur in such a context when they are not explicitly given in the stimulus. A more detailed discussion of schemata can be found elsewhere (e.g., Rumelhart \& Ortony, 1977).

Therefore, under elaborative encoding, the pattern of information activated by a trait description will include not only the memory codes that correspond to the description itself, but also representations of the default knowledge contained in the cncoding schema (Carlston, 1980). For example, upon hearing that Igor is a professor at Ivy University we may infer that Igor wears glasses and a tweed jacket, is intelligent and married, etc. The elaborations follow from instantiation of the interpreting schema. In our example this means that stereotypic knowledge about Ivy professors is applied to Igor.

It should be noted that some integration may occur during elaborative encoding. Of course, in principle, each trait in a description can be encoded within different schemata. On many an occasion, however, two or more traits will be interpreted within a single schema. As a result, rather than being represented piecemeal, these traits are chunked together and encoded as an integrated structure. We refer to this process as automatic so as to stress that integration occurs as a consequence of comprehension and takes place unintentionally, without conscious effort on the part of the individual (deliberate integration is discussed below). Generally, automatic integration will happen when two or more propositions are thematically related and there is a schema activated during comprehension that encodes this relationship (e.g., Ostrom, Lingle, Pryor, \& Geva, 1980; Smith, Adams, \& Schorr, 1978; Schul \& Burnstein, Note 1).

To induce individuals to elaboratively encode each trait in a set, they will be asked to perform an orienting task that involves semantic matching. As in physical matching, individuals indicate when they have completed reading the trait set. However, when the probe-trait is displayed, the individuals are to say whether or not it is similar in meaning (rather than identical) to one of the traits in the set. The time used to prepare for semantic matching is assumed to reflect the operations required for elaborative encoding. As in initial encoding, this process should also be sensitive to the amount of information, that is, the number of traits in 
a set. Furthermore, recall that if traits are automatically integrated, they are encoded within the same schema; otherwise, they are encoded separately. Thus, in automatic integration the encoding of one element facilitates (primes) the encoding of another element-the knowledge structure used to interpret the former is immediately available to interpret the latter. This implies that when traits are automatically integrated, they will be processed faster than when they are interpreted one at a time within separate schemata. [See similar findings in lexical priming (Meyer \& Schvaneveldt, 1971; Schvaneveldt \& Meyer, 1975).] Automatic integration is more likely when the traits in the set are consistent than when they are inconsistent. Therefore, we expect that under elaborative encoding (unlike initial encoding) processing time will vary with consistency.

Impression formation, of course, involves more than initial and elaborative encoding. On many occasions, a person has to make a judgment that takes into account a variety of traits for which no unitary interpretation immediately comes to mind. Individuals may choose one of two strategies for dealing with this problem: They can integrate the information either by discounting part of it (Abelson, 1968), or by searching for alternative schemata that are atypical (i.e., rarely activated in the situation and, hence, relatively inaccessible) but that will nonetheless accommodate all the information in a consistent fashion.

Our conjecture is that, at least in studies of impression formation, the likelihood of calling an atypical schema is higher than that of using the discounting solution. There seem to be two reasons for this: First, because our knowledge of people in real life is so rich, we are almost always able to take traits that are inconsistent in their typical context and find another context in which they would be consistent (e.g., ordinarily it is difficult to conceive of someone being "good" and "a thief," but the difficulty disappears if we imagine that this person is Robin Hood). Second, discounting strategies, at least in the laboratory, are usually inhibited by instructions to assign each trait equal weight. [See also Hendrick and Constantini (1970) for the failure of discounting mechanisms in explaining "order effects in impression formation."] But regardless of the strategy chosen, integration involves the reconciliation of inconsistencies. Hence, trait information will be represented in a more consistent fashion after integration than it had been before (Burnstein \& Schul, Note 2; Wyer, 1974).

Recall that under elaborative encoding consistent elements may be automatically integrated, whereas inconsistent elements are likely to be processed separately and represented by independent (and still inconsistent) memory codes. In other words, although inconsistencies do not have to be reconciled under elaborative encoding, they do under integration. This implies, first, that differences between the time to process consistent and inconsistent trait sets ought to be more pronounced in 
integration than in elaborative encoding (or initial encoding); second, that integration will become more time consuming as the number of tobe-integrated traits increases; and third, that the effects of increasing the number of to-be-integrated traits on processing time should be aggravated when the traits are inconsistent. The latter interaction (set size $\times$ consistency) reflects a characteristic of information structures in general and semantic network in particular, namely, that the difficulty in reconciling inconsistent information increases exponentially with the number of elements in the network.

Integration prepares the information for a final operation, the decision. This process interprets the now-integrated information within the context of an evaluative schema and translates the evaluation into a response format, such as a 6-point scale, a nod, or a smile [see Upshaws (1969) attempt to specify a model for such operations; also Parducci, 1965]. It is important to note that the decision is performed following integration, that is, after inconsistencies in the description have been minimized. The decision process, therefore, should be invariant with the initial consistency of the trait description. At the same time, it should be sensitive to the amount of information contained in the (integrated) description. The latter effect, however, is likely to be opposite to that anticipated during initial and elaborative encoding, that is, the more knowledge people have about someone they are to judge, the easier and the more rapid their decision, once this knowledge has been integrated (Posner \& Snyder, 1975). In the first study, the trait descriptions used to form an impression do not allow us to distinguish the effects of integration from those of decision. In the second study, however, trait descriptions are chosen that do permit us to examine the decision operation separately.

\section{STUDY 1}

\section{Subjects}

\section{Method}

Thirty students at the University of Michigan served as subjects. They were recruited through an advertisement in local papers and were paid $\$ 3.50$ for their participation.

\section{Stimuli}

Sets were constructed from 48 traits taken from Anderson (1968). Half of the traits were positive (scale values between 430 and 573) and half negative (scale values between 26 and 169). The sets varied in size and consistency: They contained either two traits (the small sets) or four traits (the large sets). Within a set, traits were either consistent (all positive or all negative) or inconsistent (half positive, half negative).

By taking the same 48 traits in different orders 64 sets were constructed (i.e., eight replications for each type of set). Each trait was used four times in the 64 sets, but none of the traits appeared more than once with any other trait. These will be termed the experimental sets. In a similar way, using a different group of 48 traits, we constructed 64 additional sets that served as fillers. Finally, 16 more sets were constructed for use during the practice. 


\section{Apparatus}

Subjects were seated in individual booths, equipped with a 12-in. CRT monitor, two movable buttons labeled "yes" and "no," and a six-button response box (buttons labeled from " 1 " to " 6 "). The "yes" button was moved to the subject's dominant hand. Presentation of the stimuli was controlled by a PDP-11 computer system with responses and reaction times recorded under the program control.

\section{Procedure}

Each experimental session was divided into three parts in the following order:

1. Physical matching or semantic matching. During this part subjects performed one of the first two orienting tasks. Fifteen of them were given physical matching instructions and the remaining 15 semantic matching instructions. Under physical matching a set of traits was displayed on the CRT and subjects had to press the "yes" button as soon as they had read the entire set. The set was erased from the screen following the subject's response. Immediately afterward, a probe (a single trait) was displayed. Subjects then had to press the "yes" button if the probe had appeared in the prior set, and the "no" button otherwise. Following this response, the probe was erased from the screen. There were 128 trials in this part, with an interval of $1.5 \mathrm{sec}$ between trials. Under semantic matching the instructions were similar to those for the physical matching task except for the matching criterion, that is, subjects had to press the "yes" button if the probe-trait was similar in meaning to one of the traits in the set, and "no" otherwise.

In half of the trials the probe did not match the traits in the set. These were the "experimental" sets (see above). In the remaining cases (fillers) the probe matched the set. This was done so that the probe would not prime a meaning (cf. Higgins, Rholes, \& Jones, 1977), and thereby result in a faster response during the next phase of the study.

2. Impression formation. All subjects then performed the impression formation task. During each trial a message appeared on the screen saying, "Person \#m is characterized by the following set of traits. How FAVORABLE is your impression of this person?" (m represents the trial number). Shortly afterward $(0.5 \mathrm{sec})$ the message was erased and subjects were presented with one of the experimental trait sets. They responded by pressing the button that corresponded to their impression $(1=$ very unfavorable. $.6=$ very favorable). The scale with the above labels appeared on the screen throughout. There were 64 trials in this part.

3. Consistency ratings. Following impression formation, subjects rated each experimental set for its consistency on a 6 -point scale $(1=$ very inconsistent. . $6=$ very consistent $)$. Because of time constraints, only those who had participated under physical matching instructions were used for this purpose.

To familiarize the subjects with the response mode, the first two parts of the study were preceded by 32 trials in which numbers served as stimuli. Following each "number" task, subjects had 16 warm-up trials with traits from the practice sets. Subjects received the stimuli in different random orders, and could perform at their own pace. They were instructed to respond as accurately and as quickly as they could, but not to trade accuracy for speed.

\section{Results and Discussion}

First, let us determine whether our consistency manipulation was successful. The "recognition" subjects rated each trait set for its consistency on a 6-point scale. A three-way repeated measures analysis of variance (set size $\times$ consistency $\times$ sets) was performed on these ratings. The consistency effect was highly reliable $(F(1,14)=181)$ with all positive 
or all negative trait sets being rated as more consistent than the mixed ones $\left(M ' s=4.36,3.17\right.$, respectively). ${ }^{1}$ The set-size effect failed to reach significance $(F(1,14)=1.6, p>.2)$. Finally, the interaction between set size and consistency was reliable $(F(1,14)=20.3)$, indicating that there was a greater difference between consistent and inconsistent large sets than between small ones.

During the first task, physical or semantic matching, each subject responded twice per trial. The first response, upon reading the trait set, indicated processing time for initial encoding (under physical matching) or elaborative encoding (under semantic matching). The second, made after the probe trait, merely carried through with the rationale given the subjects for the task and is used as a measure of the subject's accuracy. ${ }^{2}$ Each subject in each part provided 64 latencies, 16 in each combination of set size and consistency. Because the distribution of these scores was highly skewed (resulting in nonindependence between means and variance), we performed the following analyses on the median latencies from each combination of set size and consistency. These medians were taken from the correct responses only on the encoding tasks (part 1) and from all responses on the impression task (part 2) where accuracy was irrelevant. $^{3}$

Table 1 presents the means of the latencies. A two-way repeated measure ANOVA (set size $\times$ consistency) was performed on these scores, separately for initial encoding and for elaborative encoding. In addition, a three-way repeated measures ANOVA (set size $\times$ consistency $\times$ first task) was performed on the impression times.

Initial encoding. Subjects responded reliably faster $(F(1,14)=92.8)$ to small sets than to large sets. Also, consistent sets were processed faster than inconsistent ones $(F(1,14)=9.15)$. Finally, the interaction between set size and consistency was reliable $(F(1,14)=6.56)$, indicating that differences between consistent and inconsistent descriptions were more pronounced for large trait sets than those for small ones. This tallies with the finding (above) that large sets were perceived to be more inconsistent than small ones.

Elaborative encoding. As in initial encoding (but see below), subjects were faster with small sets than with large ones $(F(1,14)=79.4)$ and

'Unless otherwise indicated, the .05 level is used.

${ }^{2}$ On the physical match, where the matching criterion was clear cut, subjects were highly accurate (mean accuracy, 99\%), with virtually no differences due to set size or consistency. Under semantic match, however, where the matching criterion was more subjective, subjects were less accurate $(87 \%)$. As the error rate was proportional to the number of traits in the set, and independent of consistency, it will not be discussed further.

${ }^{3}$ We also performed an analysis of the median latencies taken only from those sets in which subjects were correct in the first task. There were no differences between this analysis and the analysis of impression latencies from all sets. 


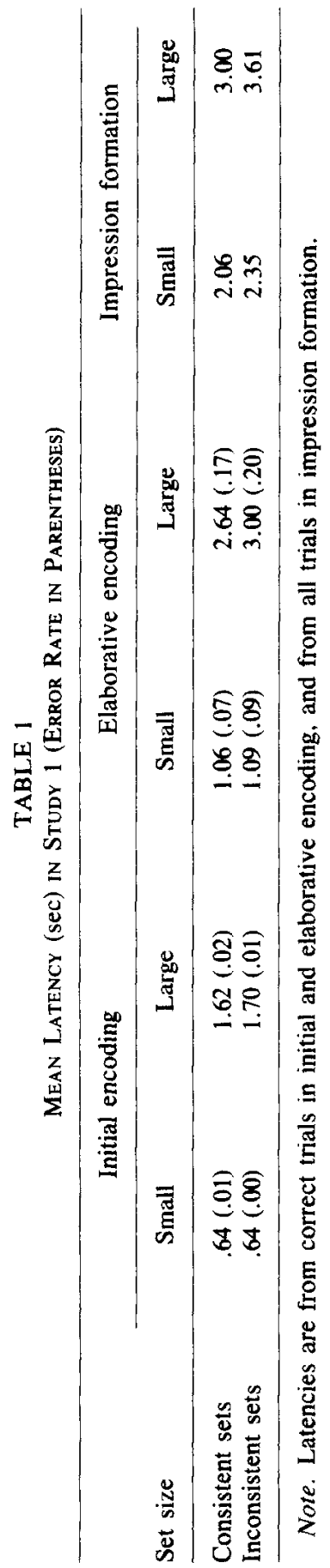


with consistent sets than with inconsistent ones $(F(1,14)=6.37)$. The interaction between set size and consistency failed to reach significance $(F(1,14)=3.13, p=.1)$.

Impression formation. Subjects who performed the first task under physical matching were reliably faster than those who performed under semantic matching $(F(1,28)=9.11)$. However, none of the interactions involving the first task reached acceptable levels of significance. Subjects formed an impression faster with small sets than with large ones $(F(1$, $28)=46.8)$, and faster with consistent sets than with inconsistent ones $(F(1,28)=20.5)$. Finally, the interaction between set size and consistency was reliable $(F(1,28)=4.26)$ and similar in pattern to that under initial encoding. As hypothesized, differences between large and small sets were more pronounced when traits were inconsistent than when they were consistent.

To summarize, both set size and consistency had reliable effects on the operations performed under the three orienting tasks. This, however, does not imply that these factors were of equal importance for performance on the different orienting tasks. For example, it is quite possible that set size explains more of the variance in initial encoding than in elaborative encoding. To assess this possibility, we computed the proportion of variance accounted for (eta square, Hays, 1963; Dwyer, 1974) by set size and consistency under cach of the threc tasks. Table 2 presents these proportions. It is quite clear that in respect to explaining the total variance, the contribution of set size decreases, and that of consistency increases as we move from initial encoding, through elaborative encoding, to impression formation.

So far we have made one assumption about the temporal order of the four processes in impression formation; that is, the decision occurs after the information has been integrated. The remaining operations, however, are likely to occur in cascade (McClelland, 1979), each starting before the other is completed (see Posner, 1978, for the temporal order of activation of semantic and physical codes). As such, these processes overlap with and feedback into each other. Suppose, however, one is willing to make the assumption that integration for the most part is performed after each piece of information is elaboratively encoded. Then one can estimate the time it takes to integrate and decide, that is, the

TABLE 2

Proportion of Variance Explained

\begin{tabular}{lcc}
\hline Processing Task & Set size & Consistency \\
\hline Initial encoding & .91 & .001 \\
Elaborative encoding & .73 & .008 \\
Impression Formation & .31 & .052 \\
\hline
\end{tabular}


integration-decision interval, by subtracting elaborative encoding time from impression formation time. A two-way repeated measures ANOVA (set size $\times$ consistency) was performed on these intervals. It revealed a reliable consistency effect $(F(1,140=5.67)$, indicating that the integration-decision interval was shorter for consistent than for inconsistent sets. Set size did not reach acceptable levels of significance $(F(1$, $14)=1.36$ ). However, the mean intervals were in marked contrast to previous analyses, namely, they were greater for snall sets than for large ones. Finally, the interaction between set size and consistency failed to reach significance.

In general, the finding on differential sensitivity to the quantity of the information and to its consistency support the partition of impression formation into initial encoding, elaborative encoding, integration, and decision. Nonetheless, there remains a problem with distinguishing between integration and decision. This is a theoretically important juncture in the impression formation process. A marked change in the effects of consistency and amount of information is presumed to occur at this point. This is to say, integration is supposed to be most sensitive to changes in consistency, whereas decision should be insensitive to this; at the same time, decision is supposed to be facilitated by increasing amounts of information, whereas integration should be hindered. However, it is difficult to devise an orienting task that will separate cleanly these two operations; once individuals deliberately integrate information, they seem automatically to proceed to make a decision.

Even though integration may not be examined independently of decision, the opposite can be attempted. In order to do so-to separate the effects of decision from integration-we tried to eliminate the need for integration by presenting "preintegrated" information. Such a procedure allows individuals to decide without having to integrate just prior to the decision. This is done in the second study by presenting a single trait (instead of the multitrait set) as a stimulus in the three orienting tasks. Here, the amount of information is varied by manipulating the associative structure of each trait, that is, its implicit information. More specifically, the traits were classified according to whether they implied a large number of other traits or only few. The former are labeled implication-rich, and the latter implication-poor.

According to our analysis, since initial encoding is minimally concerned with semantic features, it should be insensitive to whether the trait is implication-rich or implication-poor. Also, inasmuch as the implications are accessed via the activation of a single schema (i.e., they are "preintegrated"), the time to elaboratively encode an implication-poor trait should be no different than that for elaboratively encoding an implicationrich trait (Hayes-Roth, 1977; Smith et al., 1978). Integration is assumed to be bypassed since the implications of a trait are by definition already 
integrated. The decision operation, however, still ought to benefit from increasing amounts of information, here, implicit information. Therefore, the decision time for an implication-rich trait should be faster than that for an implication-poor one. Since neither initial nor elaborative encoding should be affected by the amount of implicit information associated with a trait, differences in the impression formation latencies must be due to the effects of the amount of information on the decision operation alone.

\section{STUDY 2}

\section{Method}

\section{Subjects}

Thirty students at the University of Michigan served as subjects. They were recruited through advertisement in local papers and were paid $\$ 3.50$ for their participation.

\section{Stimuli}

One hundred traits were taken from Anderson's (1968) with the following restrictions: (i) each trait consisted of a single word, (ii) containing less than 10 characters, (iii) with a frequency between 7 and 521 (Carroll, Davis, \& Richman, 1971; index d). Fifty of the traits were positive (likability values between 430 and 573), and 50 were negative (likability values between 26 and 169). Traits were presented to subjects in four different random orders. Subjects were asked to estimate the number of traits that were implied by the given trait, that is, "the number of other traits that covary (go together) with a given trait, so that when an individual has trait $A$ he is likely to have trait $B$." All ratings were made on a 9-point scale $(1=$ very few, $5=$ moderate, $9=$ very many). To validate this procedure, 42 traits ( 20 positive and 22 negative) were drawn at random from the above 100 traits. A group of 11 different subjects were asked to write down all traits implied by each of the 42 traits. For each trait we computed the mean (across subjects) of the estimated number of implied traits and the actual number of of the traits listed by the subjects. The rank-order correlation between the two was $.64(p<.01)$.

Four categories of traits were constructed by taking 10 traits that were below the mean for the estimated number of implications and 10 traits that were above the mean, separately for positive and negative traits. These traits, hereafter called experimental traits, are shown in Table 3. In addition to these, 40 additional traits were chosen according to the same criteria, and served as fillers during the first part of the experiment (see procedure). Each of the experimental traits was paired with an unrelated trait (likability values between 250 and 350). Each of the fillers was matched with a synonym (Roget's International Thesaurus, third edition). Finally, we chose 20 more traits for use during the practice.

\section{Procedure}

Subjects were run under the same conditions as those in Study 1 (i.e., individually, under computer control, with the same monitor and response buttons). Also, the first two parts of the experiment were identical in structure to those of Study 1. To begin, subjects performed either under physical matching instructions or under semantic matching instructions. Under physical matching they responded "yes" immediately after reading the first trait, and then "yes" if this trait was identical to the probe, and "no" otherwise. Under semantic matching instructions, they responded with "yes" after reading the first trait, and then "yes" if the probe was similar in meaning to the first trait, and "no" otherwise. Next, during the impression part, subjects received only the experimental traits and were instructed to form an impression about a person described by each trait. Again. 
TABLE 3

EXPERIMENTAL TratTS

\begin{tabular}{|c|c|c|c|}
\hline \multicolumn{2}{|c|}{ Implication-poor } & \multicolumn{2}{|c|}{ Implication-rich } \\
\hline Negative & Positive & Negative & Positive \\
\hline AIMLESS & COMPOSED & ANGRY & AMBITIOUS \\
\hline BORING & CULTURED & CRUEL & AMUSING \\
\hline BRAGGING & CURIOUS & DISHONEST & BRIGHT \\
\hline CARELESS & EAGER & FOOLISH & CHEERFUL \\
\hline ENVIOUS & EARNEST & HEARTLESS & CREATIVE \\
\hline HELPLESS & ETHICAL & HOSTILE & GENTLE \\
\hline JEALOUS & GRATEFUL & IMPOLITE & MATURE \\
\hline SHALLOW & LOGICAL & OFFENSIVE & SINCERE \\
\hline TOUCHY & PATIENT & SLOPPY & TRUSTING \\
\hline WEAK & PROMPT & UNKIND & WISE \\
\hline
\end{tabular}

the rating procedure used was identical to that in Study 1. In the third part, subjects were presented with the experimental traits and asked to indicate their importance in forming an opinion of a stranger. This was done on a 6-point scale $(1=$ very unimportant. . 6 $=$ very important). Finally, in the fourth part, subjects were presented with the experimental traits again, and were asked to rate them for the number of implied traits $1=$ very few. . 6 = very many).

The order of the four parts was the same for all subjects. The first two parts were preceded first by numbers tasks designed to familiarize the subjects with the response mode, and then by 20 warm-up trials with the practice traits. Subjects received the traits in a different random order and could respond at their own pace. They were instructed to respond as accurately and as fast as they could, and it was emphasized that accuracy should not be sacrificed for speed.

\section{Results and Discussion}

The rating of the amount of implicit information (part 4) was included as a manipulation check. A four-way repeated measures ANOVA [first task $\times$ trait type (implication-rich vs implication-poor) $\times$ likability $\times$ traits] was performed on these judgments. Task, likability, as well as their interactions, all failed to reach acceptable level of significance (minimum $p>.1)$. Trait-type effect was highly reliable $(F(1,28)=43.3$ ), with implication-rich traits rated as having more implications than implication-poor traits (4.14 vs 3.41 ).

As before, during the first part subjects made two responses on each trial. The first, after reading the trait description, indicated the processing time for initial encoding or for elaborative encoding. The second, after the probe trait, carried through with the rationalc given for the orienting task and was used as an accuracy measure. As in Study 1, subjects were highly accurate under physical matching instructions (see Table 4), with no difference due either to likability or trait type. Unexpectedly, under semantic matching, subjects made twice as many errors in responding to the implication-poor traits than in responding to the implication-rich 


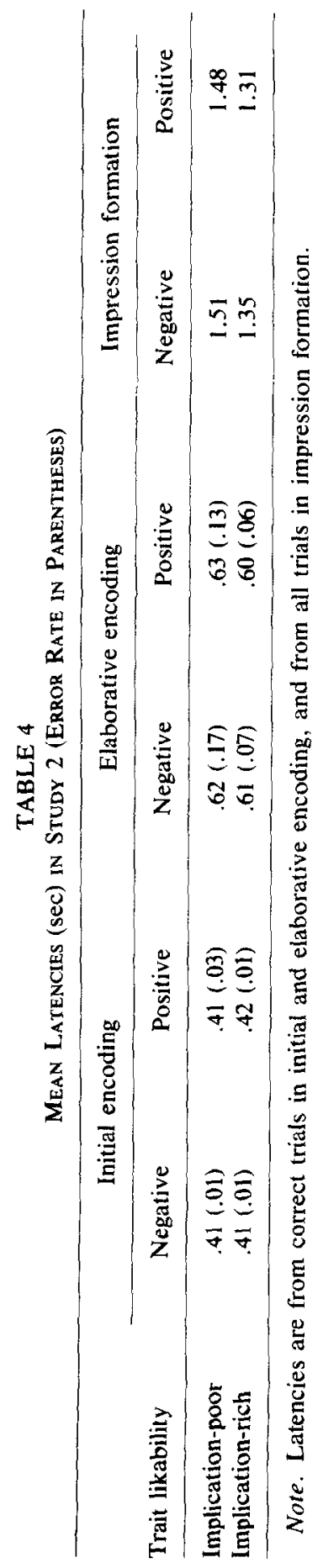


ones (see Table 4). This may indicate that the meaning of the former is less established than that of the latter, even though traits were matched for frequency.

For each combination of trait type and likability, we calculated the median latency. These medians were taken from trials with correct responses in the first part, and all trials in the impression part (also see footnote 3 ). Table 4 presents the mean latencies and error rates for the likability and trait-type factors. A two-way repeated measures ANOVA (trait type $\times$ likability) was performed on these scores under initial encoding and under elaborative encoding. In addition, a three-way repeated measures (trait type $\times$ likability $\times$ first task) was performed on the impression times. None of the main effects or interactions in the analysis of the initial encoding latencies or the elaborative encoding latencies reached acceptable levels of significance (minimum $p>.18$ ). The analysis of the impression times revealed that trait type was the only reliable effect $(F(1,28)=9.7)$, namely, impressions were formed faster with implication-rich traits than with implication-poor ones.

The lack of relationship between likability and processing time is manifested also in the low correlations between direction of judgment and the impression latency. The rank order correlation between the former and the latter was .01 , suggesting that there is no difference in operating upon positive and negative traits. This, however, does not exclude the possibility that the traits leading to an extreme impression are processed differently from those leading to a moderate impression. Judd and Kulik (1979), for example, found that judgments were made faster on items with which the person strongly agreed or strongly disagreed. We thus recoded the impressions according to their extremity, assigning 1 to the most extreme impressions ( 1 and 6 ), 2 to less extreme ( 2 and 5), and 3 to the least extreme impressions ( 3 and 4 ). Extremity was reliably associated with the amount of implicit information $\left(\chi^{2}(2)=69.66, p<\right.$ .001) such that only $42 \%$ of the implication-poor traits generated extreme impressions (1 or 6) whereas $64 \%$ of the implication-rich traits did so. This raises the unhappy possibility that the effects of implicit information were mediated by the extremity of the impression. To remove the contaminating effect of extremity, we performed a regression of the impression time on extremity. The residuals of the regression were then examined in an analysis of variance similar to the one performed on raw impression time. If the effects of implicit information on processing time were mediated by extremity, they should disappear in the analysis of the residuals. However, the pattern of results in the residuals analysis was in fact identical to that of the raw latencies. As before, neither likability nor the interaction was reliable. The trait-type effect remained reliable, albeit marginally so $(F(1,27)=3.51, p=.07)$. Thus, it seems that the effect of the amount of implicit information cannot be explained by the extremity of the judgment. 
Finally, subjects rated the traits for their importance in determining an impression. A four-way repeated measures ANOVA (first task $\times$ trait type $\times$ likability $\times$ traits) was performed on these ratings. Subjects who started with the physical matching orienting task rated the traits as more important than those who started with semantic matching $(F(1,28)=$ 6.66). Also, implication-rich traits were rated as more important in forming an impression than implication-poor ones $(F(1,28)=24.7)$. This tallies with the finding that it was easier to make a decision on the basis of the implication-rich than implication-poor traits. Likability, as well as all the interactions, failed to reach significance (minimum $p<.1$ ).

\section{GENERAL DISCUSSION}

This paper attempts to delineate the set of operations carried out in forming an impression of another person. Four functionally distinct processes were hypothesized, namely, initial encoding, elaborative encoding, integration, and decision. They were identified empirically on the basis of their differential sensitivity to the amount and consistency of information in a trait description: In Study 1 a large trait set hindered processing to a greater extent than did a small one. More importantly, this effect varied with the operation being performed. It was most pronounced during initial encoding, moderately so during elaborative encoding, and least pronounced during the integration-decision interval. Indeed, there was a tendency for the integration-decision interval to decrease as set size increased. Varying consistency produced the opposite pattern of results. Specifically, while an inconsistent trait description generally hindered processing to a larger extent than a consistent trait description, the effect was least pronounced during initial encoding, moderately so during elaborative encoding, and most pronounced during the integration-decision interval. In Study 2, the quantity of information in a description was manipulated via the associative structure, that is, the implicit knowledge activated by a trait (rather than set size). Presumably because implicit information is "preintegrated," implicationrich traits did not hinder initial or elaborative encoding any more than implication-poor traits. The decision operation, however, was performed more rapidly with implication-rich traits than with implication-poor ones. This effect seems theoretically comparable to the tendency in Study 1 for the integration-decision interval to decrease as set size increased. In any case, it strongly suggests that once information is organized by a schema, the "richer" the representation, the easier it is to arrive at a decision.

Partitioning impression formation in this manner emphasizes that the representation of a trait description in memory will depend on the operations that have been performed on such information. This has some interesting implications. To illustrate, recall our assumption that information will be stored in a discrete fashion following elaborative encoding, 
and in a unified fashion following integration. This postulate about how trait information is stored in memory inevitably leads to hypotheses about the effect of the encoding operations on accessibility. In a recent experiment (Burnstein \& Schul, Note 2) we observed that once trait information has been integrated, there is no difference between an initially consistent and an initially inconsistent description in respect to accessibility. If the traits undergo only elaborative encoding, however, the consistent description is retrieved with considerably greater accuracy than the inconsistent one. Our partitioning also makes explicit that differences in the representation can be due to differences in the extent to which the same operation is performed as well as to differences in the kind of operations. For example, consider the processing of information that is either relevant or irrelevant to a judgment. Both may undergo the same operations, but the relevant information is likely to receive more elaborative encoding than irrelevant information. As a consequence, not only should the former be more accessible than the latter, but it will also occupy a more central position in the knowledge structure upon which the judgment is based (Lingle, Geva, Ostrom, Leippe, \& Baumgardner, 1979; Schul \& Burnstein, Note 1).

Finally, our analysis makes the point that the operations performed during encoding and integration will determine the impact of the information on the judgment itself. This probably comes as no surprise. At least in the opinion change literature it is reasonably well established that information will have greater persuasive effect when it is elaborated upon; indeed, several studies indicate that the implicit information activated during elaborative encoding of a persuasive message seems to have more influence than the given information (Burnstein \& Vinokur, 1977; Caccioppo \& Pelty, 1977; Carlston, 1980; Greenwald, 1968; Tesser, 1978). Along the same lines we observed in Study 2 that traits that lend themselves to elaboration (implication-rich traits) were perceived as more important for forming an impression and actually produced more rapid as well as more extreme impressions than those that do not (implicationpoor traits). In all likelihood, therefore, the operations used to form an impression of another person are comparable to those used to fashion other opinions as well. If so, these operations should play a significant role in any general explanation of opinion formation and persuasion.

\section{REFERENCES}

Ahelson, R. P. Psychological implication. In R. P. Abelson. et al. (Eds.). Theories of cognitive consistency: A sourcebook. Chicago: Rand McNally, 1968.

Anderson, N. H. Likableness ratings of 555 personality-trait words. Journal of Personality and Social Psychology, 1968, 9, 272-279.

Burnstein, E., \& Vinikur, A. Persuasive argumentation and social comparison as determinants of attitude polarization. Journal of Experimental Social Psychology, 1977, 13, 315-332. 
Caccioppo, J. T., \& Petty, R. E. Effects of message repetition and position on cognitive response, recall, and persuasion. Journal of Personality and Social Psychology, 1977, $35,38-48$.

Carlston, D. E. The recall and use of traits and events in social inference processes. Journal of Experimental Social Psychology, 1980, 16, 303-328.

Carroll, J. B., Davis, P., \& Richman, B. Word frequency book. New York: American Heritage Co., 1971.

Craik, F. I. M., \& Tulving. E. Depth of processing and the retention of words in episodic memory. Journal of Experimental Psychology, 1975, 104, 268-294.

Dwyer, J. H. Analysis of variance and the magnitude of effects. Psychological Bulletin, 1974, 81, 731-737.

Dyer, F. N. The Stroop phenomenon and its use in study of perceptual, cognitive and response processes. Memory \& Cognition, 1973, 1, 106-120.

Ebbesen E. B. Cognitive processes in understanding ongoing behavior. In R. Hastie et al. (Eds.), Person memory: The cognitive basis of social perception. Hillsdale, N.J.: Erlbaum, 1980.

Friedman, A. Framing pictures: The role of knowledge in automatized encoding and memory for gist. Journal of Experimental Psychology, 1979, 108, 316-355.

Greenwald, A. G. Cognitive learning, cognitive response to persuasion and attitude change. In A. G. Greenwald, T. C. Brock, and T. M. Ostrom (Eds.), Psychological foundation of attitudes. New York: Academic Press, 1968.

Hastie, R., \& Carlston, D. Theoretical issues in person memory. In R. Hastie et al. (Eds.), Person memory: The cognitive basis of social perception. Hillsdale, N.J.: Erlbaum, 1980.

Hayes-Roth, B. Evolution of cognitive structures and processes. Psychological Review, $1977,84,260-278$.

Hays, W. Statistics. New York: Holt, 1963.

Hendrick, C., \& Costantini, A. Effects of varying trait inconsistency and response requirements on the primacy effect in impression formation. Journal of Personality and Social Psychology, 1970, 15, 158-164.

Higgins, T. E., Rholes, W. S., \& Jones, C. R. Category accessibility and impression formation. Journal of Experimental Social Psychology, 1977, 13, 141-154.

Judd, C. M., \& Kulik, J. A. Schematic effects of social attitudes on information processing and recall. Journal of Personality and Social Psychology, 1979, 37, 180-194.

Lingle, J. H., Geva, N., Ostrom, T. M., Leippe, M., \& Baumgardner, M. H. Thematic effects of person judgments on impression organization. Journal of Personality and Social Psychology, 1979, 37, 674-687.

McClelland, J. L. On the time relations of mental processes: An examination of systems of processes in cascade. Psychological Review, 1979, 86, 287-330.

Meyer, D. E., \& Schvaneveldt, R. W. Facilitation in recognizing pairs of words: Evidence of dependence between retrieval operations. Journal of Experimental Psychology, 1971, 90, 227-234.

Minsky, M. A framework for representing knowledge. In P. H. Winston (Ed.), The psychology of computer vision. New York: McGraw-Hill, 1975.

Newtson, D. A. Attribution and the unit of perception of ongoing behavior. Journal of Psychology and Social Psychology, 1973, 28, 23-38.

Ostrom, T. M., Lingle, J. H.. Pryor, J. B., \& Geva, N. Cognitive organization of person impressions. In R. Hastie et al. (Eds.), Person memory: The cognitive basis of social perception. Hillsdale, N.J.: Erlbaum, 1980.

Parducci, A. Range-frequency model, Psychological Review, 1965, 72, 407-418.

Posner, M. I. Coordination of internal codes. In W. G. Chase (Ed.), Visual information processing. New York: Academic Press, 1972.

Posner, M. I. Chronometric exploration of mind. New York: Erlbaum, 1978. 
Posner, M. 1., \& Keele, S. W. Decay of visual information from a single letter. Science, $1967,158,137-139$.

Posner, M. I., \& Snyder, C. R. Attention and cognitive control. In R. L. Solso (Ed.), Information processing and cognition. The Loyola Symposium. Hillsdale, N.J.: Erlbaum, 1975.

Rumelhart, D. E., \& Ortony, A. The representation of knowledge in memory. In R. C. Anderson, R. J. Spiro, \& W. E. Montague (Eds.), Schooling and the acquisition of knowledge. Hillsdale, N.J.: Erlbaum, 1977.

Schank, R. C., \& Abelson, R. P. Scripts, plans, goals, and understanding: An inquiry into human knowledge structures. Hillsdale, N.J.: Erlbaum, 1977.

Schvaneveldt. R. W., \& Meyer, D. E. Retrieval and comparison processes in semantic memory. In S. Kornblum (Ed.), Attention and performance IV. New York: Academic Press, 1973.

Smith. E. E., Adams, N., \& Schorr, D. Fact retrieval and the paradox of interference. Cognitive Psychology, 1978, 10, 438-464.

Srull, T. K., \& Wyer, R. S. The role of category accessibility in the interpretation of information about persons: Some determinants and implications. Journal of Personality and Social Psychology, 1979, 37, 1660-1662.

Tesser, A. Self-generated attitude change. In L. Berkowitz (Ed.), Advances in experimental social psychology (Vol, II). New York: Academic Press, 1978. Pp. 289-339.

Upshaw, H. S. The personal reference scale: An approach to social judgement. In L. Berkowitz (Ed.), Advances in experimental social psychology, New York: Academic Press, 1969.

Wyer, R. S. Cognitive organization and change: An information processing approach. Potomac, Md: Erlbaum, 1974.

Wyer, R. S., \& Carlston, D. E. Social cognition, inference and attribution. Hillsdale, N.J.: Erlbaum, 1979.

\section{REFERENCE NOTES}

1. Schul, Y., \& Burnstein, E. The informational basis of opinions: Memory for informative and uninformative arguments. Unpublished manuscript, University of Michigan, 1980.

2. Burnstein, E., \& Schul, Y. The informational basis of opinions: Memory for integrated and non-integrated trait descriptions. Unpublished manuscript, University of Michigan, 1980. 